\title{
Dietary $n-6$ PUFA, carbohydrate:protein ratio and change in body weight and waist circumference: a follow-up study
}

\author{
Marianne U Jakobsen ${ }^{1, *}$, Lise Madsen ${ }^{2,3}$, Claus Dethlefsen $^{4}$, Karen M Due ${ }^{4}$, \\ Jytte Halkiær ${ }^{5}$, Thorkild IA Sørensen ${ }^{6,7}$, Karsten Kristiansen $^{3}$ and Kim Overvad ${ }^{1,4}$ \\ 'Department of Public Health, Section for Epidemiology, Aarhus University, Bartholins Allé 2, DK-8000 Aarhus C, \\ Denmark: ${ }^{2}$ National Institute of Nutrition and Seafood Research, Bergen, Norway: ${ }^{3}$ Department of Biology, \\ University of Copenhagen, Copenhagen, Denmark: ${ }^{4}$ Department of Cardiology, Center for Cardiovascular \\ Research, Aalborg University Hospital, Aalborg, Denmark: ${ }^{5}$ The Danish Cancer Society Research Center, \\ Copenhagen, Denmark: ${ }^{6}$ Institute of Preventive Medicine, Bispebjerg and Frederiksberg University Hospitals - a part \\ of Copenhagen University Hospital, The Capital Region, Copenhagen, Denmark: ${ }^{7}$ Novo Nordisk Foundation Center \\ for Basic Metabolic Research, University of Copenhagen, Copenhagen, Denmark
}

Submitted 11 October 2013: Final revision received 8 June 2014: Accepted 26 June 2014: First published online 27 August 2014

\begin{abstract}
Objective: To investigate the association between the intake of $n$ - 6 PUFA and subsequent change in body weight and waist circumference at different levels of the carbohydrate:protein ratio.

Design: Follow-up study with anthropometric measurements at recruitment and on average 5.3 years later. Dietary intake was determined at recruitment by using an FFQ that was designed for the study and validated. We applied linear regression models with 5-year change in weight or waist circumference as outcome and including a two-way interaction term between $n$ - 6 PUFA and carbohydrate intakes, lower-order terms, protein intake, long-chain $n$-3 PUFA intake and other potential confounders. Due to adjustment for intake of protein, levels of carbohydrate indirectly reflect levels of the carbohydrate:protein ratio.

Setting: Diet, Cancer and Health follow-up study, Denmark.

Subjects: Women and men ( $n$ 29 152) aged 55 years.

Results: For a high intake of $n-6$ PUFA (6.9\% of energy) $v$. a low intake of $n-6$ PUFA (3.4\% of energy), the difference in 5-year weight change was $-189.7 \mathrm{~g}$ $(95 \% \mathrm{CI}-636.8,257.4 \mathrm{~g})$ at a low carbohydrate:protein ratio and $-86.7 \mathrm{~g}(95 \% \mathrm{CI}$ $-502.9,329.6 \mathrm{~g})$ at a high carbohydrate:protein ratio; the differences in 5-year waist circumference change were $0.26 \mathrm{~cm}(95 \% \mathrm{CI}-0.47,0.98 \mathrm{~cm})$ and $-0.52 \mathrm{~cm}$ $(95 \% \mathrm{CI}-1 \cdot 19,0 \cdot 15 \mathrm{~cm})$, respectively. Inclusion of the dietary glycaemic index did not change the results.

Conclusions: No consistent associations between the intake of $n-6$ PUFA and change in body weight or waist circumference at different levels of the carbohydrate:protein ratio were observed.
\end{abstract}

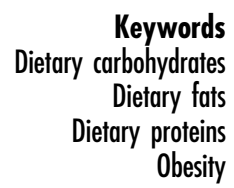

BMI and waist circumference are proxy measures of overall body fat mass and abdominal fat mass, respectively, and strong predictors of obesity-related mortality ${ }^{(1)}$. Studies on the evolutionary aspects of diet indicate that major changes have taken place in the Western diet, particularly during the last 100 years; changes that correlate with the increasing prevalence of obesity ${ }^{(2)}$. Today, more than $85 \%$ of the total intake of $n-6$ and $n-3$ PUFA is $n-6$ PUFA $^{(3)}$. In the USA, the estimated per capita consumption of soyabean oil, the major source of the n-6 PUFA linoleic acid (18: 2n-6), has increased more than 1000-fold from 1909 to $1999^{(4)}$; thus, the availability of $18: 2 n-6$ increased from $2 \cdot 8 \%$ to $7 \cdot 2 \%$ of energy during the 20th century. Interesting, the increase in the availability of soyabean oil paralleled the increase in the prevalence of obesity ${ }^{(2)}$. Likewise, the availability of sugars was also positively correlated with obesity whereas energy was not $^{(2)}$. Of note, in mice, increasing $18: 2 n-6$ from $1 \%$ to $8 \%$ of energy in the feed, reflecting the 20th century increase in availability of $18: 2 n-6$, promoted obesity ${ }^{(2)}$. Still, in man, the effect of $n-6$ PUFA on obesity development has not been investigated in detail.

The findings from the mouse experiment by Alvheim et $a l{ }^{(2)}$ are in line with other animal experiments which 
have reported that feed high in $n-6$ PUFA increased adipose tissue ${ }^{(5,6)}$. In contrast, other animal experiments have reported that feed high in n-6 PUFA decreased adipose tissue mass ${ }^{(7,8)}$. In animals, the apparent conflicting findings may be explained by different background diets, as Madsen et $a l .{ }^{(9)}$ have shown that the effect of $n$-6 PUFA on adipose tissue mass is determined by the balance between carbohydrates and proteins. In mice, when the carbohydrate:protein ratio was high, also the plasma insulin:glucagon ratio was high and high $n$-6 PUFA intake increased adipose tissue mass. Conversely, when the carbohydrate:protein ratio was low, also the plasma insulin:glucagon ratio was low and high $n-6$ PUFA intake decreased adipose tissue mass. If the background diet, via changes in the insulin:glucagon ratio, also determines the effect of $n$ - 6 PUFA on adipose tissue mass in man, both the amount and type of dietary carbohydrates may be of importance.

Based on findings from animal experiments, we hypothesized that in man a high intake of $n-6$ PUFA is associated with subsequent loss in body weight and decreased waist circumference at a low carbohydrate:protein ratio but associated with subsequent gain in body weight and increased waist circumference at a high carbohydrate: protein ratio. We investigated the association between the intake of $n-6$ PUFA and subsequent change in body weight and waist circumference at different levels of the carbohydrate:protein ratio. The dietary glycaemic index was also included.

\section{Methods}

\section{Study design and population}

The present study was based on data from the Danish follow-up study Diet, Cancer and Health. The primary aim of the Diet, Cancer and Health is to investigate the associations between foods and nutrients, lifestyle and the incidence of cancer and other chronic diseases ${ }^{(10)}$. Between December 1993 and May 1997, 160725 women and men were invited by mail to participate in the Diet, Cancer and Health. The criteria for invitation were as follows: age between 50 and 64 years, born in Denmark and no previous cancer diagnosis registered in the Danish Cancer Registry. All persons fulfilling these criteria and living in the greater Copenhagen or Aarhus areas were invited. With the invitation, a validated 192-item semiquantitative FFQ was enclosed $^{(11,12)}$. At the study centre, participants filled in a lifestyle questionnaire and a physical examination was conducted including anthropometric measurements. The self-administered questionnaires were scanned and checked by laboratory technicians. In total, 57053 (35\%) participants were recruited. Follow-up information on anthropometry was collected about 5 years after recruitment. A detailed description of the study design and measurement procedures has been given by
Tjønneland et al. ${ }^{(10)}$. The study was conducted according to the guidelines laid down in the Declaration of Helsinki and all procedures involving human subjects were approved by the Copenhagen and Aarhus Ethical Committees. Written informed consent was obtained from all participants.

\section{Dietary assessments}

The participants filled in the 192-item semi-quantitative FFQ, which was designed for the Diet, Cancer and Health ${ }^{(11)}$ and validated against two $7 \mathrm{~d}$ weighed diet records ${ }^{(12)}$. Intake of foods and alcohol consumption were reported as the average amount during the past year. Calculation into daily nutrient intakes was done using the FoodCalc program (http://www.ibt.ku.dk/jesper/foodcalc), which is based on Danish food composition tables $1996^{(13)}$. Derived exposure measure was intake of $n-6$ PUFA (the sum of $18: 2 n-6$ and arachidonic acid (20:4n-6)) expressed as percentage of energy (not including energy intake derived from alcohol consumption). Glycaemic index values obtained from published lists expressed as a percentage of the value for white bread were assigned to the carbohydrate-containing foods reported in the $\mathrm{FFQ}^{(14)}$. For each participant, the dietary glycaemic index was calculated by adding up the products of the amount of glycaemic carbohydrates (total carbohydrates minus fibre) of each food (i.e. the proportion of glycaemic carbohydrates), the amount of that food eaten daily and its glycaemic index value, and then dividing this total by the total amount of glycaemic carbohydrates in one day.

\section{Anthropometric measurements}

At recruitment, technicians measured participants' height, weight and waist circumference. Participants' height was measured to the nearest $0.5 \mathrm{~cm}$ when standing without wearing shoes. Weight was measured to the nearest $0 \cdot 1 \mathrm{~kg}$ using digital scales, with participants wearing light underwear. Waist circumference was measured to the nearest $0.5 \mathrm{~cm}$ using a measuring tape at the narrowest horizontal circumference between the lower rib and the iliac crest (the natural waist) when standing. In cases of indeterminate waist narrowing, waist circumference was measured half way between the lower rib and the iliac crest.

At follow-up, information on weight and waist circumference was collected through a self-administered questionnaire. A measuring tape was provided with the questionnaire and to ease the identification of site of measurement, the participants were asked to measure waist circumference at the level of the umbilicus. A validation study within this cohort has shown that the selfmeasured waist circumference at the level of the umbilicus is usable as a proxy for the technician-measured waist circumference at the natural waist in regression analyses where waist circumference and BMI at recruitment were included as covariates ${ }^{(15)}$. 
The outcome measures were the difference between weight at recruitment and weight at follow-up and the difference between waist circumference at recruitment and waist circumference at follow-up expressed as 5-year change. BMI was calculated as weight in kilograms divided by the square of height in metres.

\section{Covariates}

Information on education, smoking status and leisure-time physical activity was obtained from the lifestyle questionnaire at baseline. Length of education was collected in predefined categories ( $<8,8-10$ and $>10$ years). Smoking status was reported as: never; former; current; the number of cigarettes, cigars, cheroots and tobacco pipes smoked per day; and duration of smoking in years. Current tobacco consumption was calculated in grams per day by summing the reported numbers per day using conversion factors of 1.0 for cigarettes, 4.5 for cigars and 3.0 for cheroots and tobacco pipes. Leisure-time physical activity during the past year was assessed from questions about the average number of hours per week spent on six types of activities (walking, gardening, housework, home maintenance, sports and biking) during summer and winter. Leisure-time physical activity was calculated as hours per week spent on sports and biking (including as a means of transport).

\section{Exclusion criteria}

Participants with a cancer diagnosis that was not at the time of invitation registered in the Danish Cancer Registry (due to processing delay) were later excluded, as were participants registered with a cancer diagnosis during follow-up. Furthermore, participants whose questionnaires were incomplete and participants with self-reported diabetes mellitus or registered with a CVD or chronic obstructive pulmonary disease diagnosis at recruitment or during follow-up were also excluded. The rationale for exclusion of participants with diabetes mellitus, cancer, CVD or chronic obstructive pulmonary disease was potential changes in dietary habits and anthropometry as a result of diagnosis and treatment, together with the unclear timing of change in anthropometry.

\section{Statistical analyses}

The characteristics of the study population were described by medians and $80 \%$ central range or by percentages.

The interplay between the intake of $n-6$ PUFA, the carbohydrate:protein ratio and subsequent change in weight or waist circumference was investigated by using a linear regression model with 5-year weight change (g) or waist circumference change $(\mathrm{cm})$ as outcome and including a two-way interaction term between quintiles of $n-6$ PUFA (\% of energy) and quintiles of glycaemic carbohydrates (\% of energy) as well as lower-order terms among all participants and separately for women and men. The covariates were sex (among all participants), age (years), BMI at recruitment $\left(\mathrm{kg} / \mathrm{m}^{2}\right)$, waist circumference at recruitment $(\mathrm{cm}$, in the analyses of waist circumference only), education ( $<8,8-10$ and $>10$ years), smoking status (never, former and currently smoking 1-14, 15-24 or $\geq 25$ g tobacco/d), leisure-time physical activity (h/week), alcohol consumption $(\mathrm{g} / \mathrm{d})$, and intakes of proteins (\% of energy), long-chain $n-3$ PUFA (the sum of $20: 5 n-3$, $22: 5 n-3$ and $22: 6 n-3, \mathrm{~g} / \mathrm{d})$ and energy $(\mathrm{kJ} / \mathrm{d})$. Adjustment for continuous variables was done using restricted cubic spline regression. Due to adjustment for intake of proteins, quintiles of carbohydrates indirectly reflect quintiles of the carbohydrate:protein ratio. Supplementary analyses including intake of $n-6$ PUFA as linear effect were also conducted.

In further analyses, we included a three-way interaction between quintiles of $n-6$ PUFA, quintiles of carbohydrates and quintiles of dietary glycaemic index, as well as lowerorder terms and covariates as described above. The differences in absolute weight changes and waist circumference changes with low $n$-6 PUFA intake (first quintile) as the reference group were calculated. The results in subgroups from analyses stratified by sex were not different. Consequently, estimates are reported for the two sexes combined.

Reduction of lean mass is a primary body composition change associated with ageing. Therefore, we repeated the analyses after excluding participants 60 years or older at baseline. Under- and over-reporting of energy intake was taken into account in additional analyses where participants in the lowest and highest $1 \%$ of the sex-specific distribution of energy intake were excluded. Implausible changes in weight or waist circumference were taken into account by further exclusion of participants in the lowest and highest $1 \%$ of the sex-specific distribution of 5-year changes in weight or waist circumference.

Data analyses were performed using the Stata statistical software package release $11 \cdot 2$.

\section{Results}

Among the 57053 women and men recruited, 569 participants with a cancer diagnosis not registered in the Danish Cancer Registry at the time of invitation (due to processing delay) were later excluded. Between the examination at recruitment and the time of invitation to the follow-up examination, 1778 participants had died and 460 participants had emigrated or disappeared. Among the 54246 participants eligible for invitation to follow-up, 5865 did not respond and 2858 did not want to participate. The participation in the follow-up was thus $81 \%$. Among the 45523 participants at follow-up, 1708 participants with incomplete questionnaires were excluded. Furthermore, 
participants with self-reported diabetes mellitus or registered with a CVD or chronic obstructive pulmonary disease diagnosis at recruitment or during follow-up, as well as participants registered with a cancer diagnosis during follow-up, were also excluded ( $n$ 14663). The final population consisted of 29152 persons (56\% women).

Characteristics of the study population are shown in Table 1 . The median follow-up time was $5 \cdot 3$ years $(80 \%$ central range: $5 \cdot 1,5 \cdot 7$ years).
Table 2 shows the difference and 95\% confidence interval of 5-year body weight change according to intake of $n-6$ PUFA at different levels of intake of carbohydrates. Due to adjustment for intake of proteins, levels of carbohydrates indirectly reflect levels of the carbohydrate:protein ratio. For a high intake of $n-6$ PUFA $v$. a low intake of $n$-6 PUFA, the difference in 5-year weight change was $-189.7 \mathrm{~g}(95 \% \mathrm{CI}-636 \cdot 8,257.4 \mathrm{~g})$ at a low carbohydrate: protein ratio and $-86.7 \mathrm{~g}(95 \% \mathrm{CI}-502.9,329.6 \mathrm{~g})$ at a

Table 1 Characteristics of the study population*: Diet, Cancer and Health follow-up study, Denmark

\begin{tabular}{|c|c|c|c|c|c|c|}
\hline & \multicolumn{2}{|c|}{ All participants } & \multicolumn{2}{|c|}{ Women } & \multicolumn{2}{|c|}{ Men } \\
\hline & $\begin{array}{l}\text { Median or } \\
\%\end{array}$ & $\begin{array}{l}80 \% \text { central } \\
\text { range }\end{array}$ & $\begin{array}{l}\text { Median or } \\
\%\end{array}$ & $\begin{array}{l}80 \% \text { central } \\
\text { range }\end{array}$ & $\begin{array}{l}\text { Median or } \\
\%\end{array}$ & $\begin{array}{l}80 \% \text { central } \\
\text { range }\end{array}$ \\
\hline Age (years) & 55 & 51,63 & 56 & 51,63 & 55 & 51,62 \\
\hline Postmenopausal (\%) & - & - & 57 & - & - & - \\
\hline Height $(\mathrm{m})$ & $1 \cdot 70$ & $1.59,1.82$ & 1.64 & $1.57,1.72$ & $1 \cdot 77$ & $1.69,1.86$ \\
\hline Weight (kg) & $73 \cdot 1$ & $58 \cdot 1,91 \cdot 5$ & $66 \cdot 5$ & $55 \cdot 4,82 \cdot 6$ & $80 \cdot 9$ & $68 \cdot 6,96.5$ \\
\hline $\mathrm{BMI}\left(\mathrm{kg} / \mathrm{m}^{2}\right)$ & 25 & 21,30 & 25 & 21,30 & 26 & 22,30 \\
\hline Waist circumference (cm) & 87 & 72,102 & 79 & 69,95 & 94 & 84,106 \\
\hline 5-year body weight change (g) & -97 & $-4678,4325$ & -258 & $-4906,4190$ & 89 & $-4348,4483$ \\
\hline $\begin{array}{l}\text { 5-year waist circumference change } \\
\text { (cm) }\end{array}$ & $4 \cdot 6$ & $-2 \cdot 9,13 \cdot 8$ & $6 \cdot 2$ & $-1.9,16.6$ & $2 \cdot 8$ & $-3.9,9.5$ \\
\hline More than 10 years of education (\%) & 23 & - & 21 & - & 27 & - \\
\hline Current smoker (\%) & 30 & - & 27 & - & 34 & - \\
\hline Leisure-time physical activity (h/week) & 2.5 & $0.0,8.0$ & $3 \cdot 0$ & $0.0,8.0$ & 2.5 & $0.0,8.0$ \\
\hline Alcohol consumption $(\mathrm{g} / \mathrm{d})$ & $12 \cdot 9$ & $1 \cdot 8,44 \cdot 2$ & $9 \cdot 9$ & $1 \cdot 3,35 \cdot 6$ & $19 \cdot 3$ & $4 \cdot 7,59 \cdot 6$ \\
\hline Energy $(\mathrm{kJ} / \mathrm{d})$ & 8447 & 5841,11829 & 7653 & 5418,10565 & 9460 & 6866,12841 \\
\hline Total fat (\% of energy) & $36 \cdot 7$ & $29 \cdot 5,42 \cdot 4$ & $35 \cdot 5$ & $28.5,41.4$ & $38 \cdot 0$ & $31 \cdot 4,43.4$ \\
\hline $18: 2 n-6(g / d)$ & $10 \cdot 7$ & $6 \cdot 2,17 \cdot 7$ & $9 \cdot 5$ & $5 \cdot 6,15 \cdot 6$ & $12 \cdot 3$ & $7 \cdot 41,19.6$ \\
\hline $20: 4 n-6(g / d)$ & 0.1 & $0 \cdot 1,0 \cdot 2$ & $0 \cdot 1$ & $0.0,0.2$ & $0 \cdot 1$ & $0.1,0.2$ \\
\hline$n-6$ PUFA $(g / d) \dagger$ & $10 \cdot 8$ & $6 \cdot 3,17 \cdot 8$ & $9 \cdot 6$ & $5 \cdot 7,15 \cdot 8$ & $12 \cdot 5$ & $7 \cdot 5,19 \cdot 8$ \\
\hline$n-6$ PUFA (\% of energy) & $4 \cdot 8$ & $3.4,6.9$ & $4 \cdot 7$ & $3 \cdot 3,6 \cdot 9$ & 4.9 & $3 \cdot 5,7 \cdot 0$ \\
\hline Long-chain $n$-3 PUFA (g/d)‡ & 0.6 & $0.3,1 \cdot 2$ & 0.6 & $0.3,1 \cdot 1$ & 0.7 & $0.3,1.3$ \\
\hline $\begin{array}{l}\text { Glycaemic carbohydrates ( } \% \text { of } \\
\text { energy) }\end{array}$ & $45 \cdot 0$ & $38 \cdot 5,52 \cdot 5$ & $46 \cdot 1$ & $39 \cdot 6,53 \cdot 6$ & $43 \cdot 6$ & $37 \cdot 4,50 \cdot 6$ \\
\hline Proteins (\% of energy) & $18 \cdot 4$ & $15 \cdot 3,21 \cdot 7$ & $18 \cdot 3$ & $15 \cdot 2,21 \cdot 7$ & $18 \cdot 4$ & $15 \cdot 5,21 \cdot 7$ \\
\hline Carbohydrate:protein ratio & 2.5 & $1 \cdot 9,3 \cdot 2$ & 2.5 & $1.9,3 \cdot 3$ & $2 \cdot 4$ & $1 \cdot 8,3 \cdot 1$ \\
\hline Dietary glycaemic index & $86 \cdot 6$ & $79 \cdot 7,93 \cdot 6$ & $85 \cdot 1$ & $78 \cdot 5,91 \cdot 7$ & $88 \cdot 7$ & $82 \cdot 1,95 \cdot 0$ \\
\hline
\end{tabular}

${ }^{*} n 29152$ for all participants; $n 16248$ for women; $n 12904$ for men.

†The sum of intakes of $18: 2 n-6$ and $20: 4 n-6$.

$\ddagger$ The sum of intakes of $20: 5 n-3,22: 5 n-3$ and $22: 6 n-3$.

Table 2 Difference and $95 \%$ confidence interval of 5-year body weight change $(\mathrm{g})$ according to intake of $n$-6 PUFA at different levels of intake of carbohydrates* ${ }^{*} \dagger$ : Diet, Cancer and Health follow-up study, Denmark

\begin{tabular}{|c|c|c|c|c|c|c|}
\hline \multirow[b]{3}{*}{ Carbohydrates } & \multicolumn{6}{|c|}{$n-6$ PUFA } \\
\hline & \multicolumn{2}{|c|}{$\begin{array}{c}\text { Low (3.4\% of energy) } \ddagger \\
\text { (1st quintile) }\end{array}$} & \multicolumn{2}{|c|}{$\begin{array}{c}\text { Medium ( } 4.8 \% \text { of energy) } \\
\text { (3rd quintile) }\end{array}$} & \multicolumn{2}{|c|}{$\begin{array}{c}\text { High (6.9\% of energy) } \\
\text { (5th quintile) }\end{array}$} \\
\hline & $\beta$ & $95 \% \mathrm{Cl}$ & $\beta$ & $95 \% \mathrm{Cl}$ & $\beta$ & $95 \% \mathrm{Cl}$ \\
\hline $\begin{array}{l}\text { Low (38.5\% of energy) (1st quintile) } \\
\text { Medium ( } 45.0 \% \text { of energy) (3rd quintile) } \\
\text { High ( } 52.5 \% \text { of energy) (5th quintile) }\end{array}$ & $\begin{array}{l}\text { Reference } \\
\text { Reference } \\
\text { Reference }\end{array}$ & $\begin{array}{l}- \\
-\end{array}$ & $\begin{array}{r}-19 \cdot 0 \\
-165 \cdot 3 \\
562 \cdot 2\end{array}$ & $\begin{array}{r}-489 \cdot 0,451 \cdot 0 \\
-529 \cdot 8,199 \cdot 2 \\
230 \cdot 8,893 \cdot 5\end{array}$ & $\begin{array}{r}-189.7 \\
-22 \cdot 2 \\
-86.7\end{array}$ & $\begin{array}{l}-636 \cdot 8,257 \cdot 4 \\
-395 \cdot 0,350 \cdot 7 \\
-502 \cdot 9,329 \cdot 6\end{array}$ \\
\hline \multicolumn{7}{|c|}{$\begin{array}{l}{ }^{*} n 462 \text { for low carbohydrates and low } n-6 \text { PUFA; } n 1193 \text { for low carbohydrates and medium } n-6 \text { PUFA; } n 1847 \text { for low carbohydrates and high } n-6 \text { PUFA; } n 989 \\
\text { for medium carbohydrates and low } n-6 \text { PUFA; } n 1253 \text { for medium carbohydrates and medium } n-6 \text { PUFA; } n 1146 \text { for medium carbohydrates and high } n-6 \text { PUFA, } \\
n 2320 \text { for high carbohydrates and low } n-6 \text { PUFA; } n 939 \text { for high carbohydrates and medium } n-6 \text { PUFA; } n 519 \text { for high carbohydrates and high } n-6 \text { PUFA. } \\
\text { †The interplay between intake of } n-6 \text { PUFA, carbohydrate:protein ratio and subsequent change in weight was investigated by using a linear regression model } \\
\text { with } 5 \text {-year weight change as outcome and including a two-way interaction term between quintiles of } n-6 \text { PUFA and quintiles of carbohydrates as well as lower- } \\
\text { order terms. The covariates were sex, age, BMI at recruitment, education, smoking status, leisure-time physical activity, alcohol consumption, and intakes of } \\
\text { proteins, long-chain } n-3 \text { PUFA and energy. Due to adjustment for intake of proteins, quintiles of carbohydrates indirectly reflect quintiles of the carbohydrate: } \\
\text { protein ratio. } \\
\ddagger \text { Median intake (all such values). }\end{array}$} \\
\hline
\end{tabular}


Table 3 Difference and $95 \%$ confidence interval of 5-year waist circumference change $(\mathrm{cm})$ according to intake of $n$-6 PUFA at different levels of intake of carbohydrates ${ }^{\star}, \dagger$ : Diet, Cancer and Health follow-up study, Denmark

$n-6$ PUFA

\begin{tabular}{|c|c|c|c|c|c|c|}
\hline \multirow[b]{3}{*}{ Carbohydrates } & \multirow{2}{*}{\multicolumn{2}{|c|}{$\begin{array}{c}\text { Low (3.4\% of energy) } \ddagger \\
\text { (1st quintile) }\end{array}$}} & & & & \\
\hline & & & \multicolumn{2}{|c|}{$\begin{array}{c}\text { Medium (4.8\% of energy) } \\
\text { (3rd quintile) }\end{array}$} & \multicolumn{2}{|c|}{$\begin{array}{c}\text { High ( } 6.9 \% \text { of energy) } \\
\text { (5th quintile) }\end{array}$} \\
\hline & $\beta$ & $95 \% \mathrm{Cl}$ & $\beta$ & $95 \% \mathrm{Cl}$ & $\beta$ & $95 \% \mathrm{Cl}$ \\
\hline Low (38.5 \% of energy) (1st quintile) & Reference & - & 0.42 & $-0.34,1.18$ & 0.26 & $-0.47,0.98$ \\
\hline Medium ( $45.0 \%$ of energy) (3rd quintile) & Reference & - & 0.16 & $-0.43,0.75$ & 0.29 & $-0.32,0.89$ \\
\hline High (52.5\% of energy) (5th quintile) & Reference & - & 0.47 & $-0.06,1.01$ & -0.52 & $-1 \cdot 19,0 \cdot 15$ \\
\hline
\end{tabular}

${ }^{*} n 462$ for low carbohydrates and low $n$-6 PUFA; $n 1193$ for low carbohydrates and medium $n-6$ PUFA; $n$ 1847 for low carbohydrates and high $n$ - 6 PUFA; $n$ 989 for medium carbohydrates and low $n$-6 PUFA; $n 1253$ for medium carbohydrates and medium $n-6$ PUFA; $n 1146$ for medium carbohydrates and high $n$ - 6 PUFA; $n 2320$ for high carbohydrates and low $n-6$ PUFA, $n 939$ for high carbohydrates and medium $n-6$ PUFA; $n 519$ for high carbohydrates and high $n-6$ PUFA. †The interplay between intake of $n-6$ PUFA, carbohydrate:protein ratio and subsequent change in waist circumference was investigated by using a linear regression model with 5-year waist circumference change as outcome and including a two-way interaction term between quintiles of $n$ - 6 PUFA and quintiles of carbohydrates as well as lower-order terms. The covariates were sex, age, BMI at recruitment, waist circumference at recruitment, education, smoking status, leisure-time physical activity, alcohol consumption, and intakes of proteins, long-chain $n$-3 PUFA and energy. Due to adjustment for intake of proteins, quintiles of carbohydrates indirectly reflect quintiles of the carbohydrate:protein ratio. †Median intake (all such values).

high carbohydrate:protein ratio (Table 2); the differences in 5-year waist circumference change were $0.26 \mathrm{~cm}(95 \%$ $\mathrm{CI}-0.47,0.98 \mathrm{~cm})$ and $-0.52 \mathrm{~cm}(95 \% \mathrm{CI}-1 \cdot 19,0.15 \mathrm{~cm})$, respectively (Table 3 ). Including intake of $n-6$ PUFA as linear effects revealed similar results to the categorical analyses (data not shown).

Information on the difference and 95\% confidence interval of 5-year body weight change according to intake of $n-6$ PUFA when the carbohydrate:protein ratio and the dietary glycaemic index were taken into account can be found in the online supplementary material, Table 1 and information on the difference and $95 \%$ confidence interval of 5-year waist circumference change according to intake of $n-6$ PUFA when the carbohydrate:protein ratio and the dietary glycaemic index were taken into account can be found in the online supplementary material, Table 2 . For a high intake of $n$-6 PUFA $v$. a low intake of $n-6$ PUFA at a low dietary glycaemic index, the difference in 5-year weight change was $-208.5 \mathrm{~g}(95 \% \mathrm{CI}-1109 \cdot 0,691.9 \mathrm{~g})$ at a low carbohydrate:protein ratio and $154.9 \mathrm{~g}$ (95\% CI $-717 \cdot 6,1027.5 \mathrm{~g}$ ) at a high carbohydrate:protein ratio; for a high intake of $n-6$ PUFA $v$. a low intake of $n-6$ PUFA at a high dietary glycaemic index, the differences in 5-year weight change were $-1367.2 \mathrm{~g} \quad(95 \%$ CI $-2396 \cdot 8$, $-337.7 \mathrm{~g})$ and $-559.3 \mathrm{~g}(95 \% \mathrm{CI}-1566.3,447.7 \mathrm{~g})$, respectively (supplementary material, Table 1 ). For a high intake of $n-6$ PUFA $v$. a low intake of $n-6$ PUFA at a low dietary glycaemic index, the difference in 5-year waist circumference change was $0.44 \mathrm{~cm} \quad(95 \%$ CI -1.02 , $1.89 \mathrm{~cm})$ at a low carbohydrate:protein ratio and $-0.32 \mathrm{~cm}$ $(95 \% \mathrm{CI}-1.73,1.09 \mathrm{~cm})$ at a high carbohydrate:protein ratio; for a high intake of $n-6$ PUFA $v$. a low intake of n- 6 PUFA at a high dietary glycaemic index, the differences in 5-year waist circumference change were $0.21 \mathrm{~cm}(95 \% \mathrm{CI}$ $-1.46,1.88 \mathrm{~cm})$ and $-1.42 \mathrm{~cm}(95 \% \mathrm{CI}-3.05,0.21)$, respectively (supplementary material, Table 2 ).

Exclusion of participants 60 years or older at recruitment did not change the results (data not shown). Also, neither exclusion of participants in the lowest and highest $1 \%$ of the sex-specific distribution of energy intake at recruitment nor further exclusion of participants in the lowest and highest $1 \%$ of the sex-specific distribution of 5-year changes in weight or waist circumference changed the results (data not shown).

\section{Discussion}

In the present study, no consistent associations between the intake of $n$ - 6 PUFA and change in body weight or waist circumference at different levels of the carbohydrate: protein ratio were observed. We used data from a large follow-up study, which allowed us to investigate the interplay between dietary n-6 PUFA, carbohydrate:protein ratio and subsequent change in weight and waist circumference among women and men within the range of $n$-6 PUFA intake in the general population. Categorical analysis was used to maximize the exposure contrast, which caused loss of statistical power and thus wide confidence intervals. However, linear analyses revealed similar results (data not shown). Exclusions were primarily due to non-response at follow-up. However, only minor differences between the baseline intake of $n-6$ PUFA among the study population and the baseline intake of $n-6$ PUFA among the non-participants were observed (data not shown). Thus, selection bias is unlikely to have affected the results. A potential source of random measurement error arises from dietary self-reporting methods. Generally, random measurement error leads to underestimation of the true association and to loss of statistical power. However, dietary intake was determined by using an FFQ, which may reflect the habitual eating pattern. The glycaemic index of foods is only one factor that determines the overall glycaemic impact of the diet; other factors include the amounts of carbohydrates, fatty acids and proteins. Furthermore, the glycaemic index of foods can 
change depending on the preparation method. In the current study we controlled for amounts of carbohydrates by default; thus taking into account both the amount and the type of carbohydrates. At recruitment, anthropometry was measured by trained staff but at follow-up, information on anthropometry was self-measured by the participants. An overall net weight loss during follow-up was found in the study. This may be due to the age of the study population and/or due to under-reporting of follow-up weight. Most likely, however, the reporting of follow-up weight and waist circumference was not related to the exposure to $n-6$ PUFA. Thus, information bias is of limited concern. Detailed information on the potential confounders reduces the possibility of residual confounding. However, confounding from unknown risk factors for development of obesity not taken into account is possible.

Experiments in mice have shown that the balance between carbohydrates and proteins in the feed may determine the effect of intake of $n$ - 6 PUFA on adipose tissue mass ${ }^{(9)}$; thus, high $n$-6 PUFA intake decreased adipose tissue mass when combined with a low carbohydrate:protein ratio, but increased adipose tissue mass when combined with a high carbohydrate:protein ratio. In the present human study, no consistent associations between the intake of $n-6$ PUFA at a low, medium or high carbohydrate:protein ratio and change in body weight or waist circumference were observed. This may be due to lower levels of intake of $n$ - 6 PUFA ( $80 \%$ central range: $3 \cdot 4$, $6.9 \%$ of energy), higher levels of intake of carbohydrates ( $80 \%$ central range: $38 \cdot 5,52 \cdot 5 \%$ of energy) and/or limited exposure contrasts. Generally, animal experiments used high doses of $n-6$ PUFA $^{(5-8)}$, such as $22 \%$ of energy from n-6 PUFA in the mouse experiment by Madsen et al. ${ }^{(9)}$ (Lise Madsen, personal communication, 2013), but did not provide information on lower intakes compatible with intakes in the general population. However, in the mouse experiment by Alvheim et al. ${ }^{(2)}$ increasing 18 : $2 n-6$ from $1 \%$ to $8 \%$ of energy in the feed was sufficient to promote obesity. In the mouse experiment by Madsen et al. ${ }^{(9)}$, the intake of carbohydrates constituted $8 \%$ of the energy intake in the low-carbohydrate group and $37 \%$ in the high-carbohydrate group; intakes below the 10th percentile intake in the present study. The finding of the importance of the carbohydrate:protein ratio in the mouse experiment $^{(9)}$ is in line with the results from a large European human trial on weight-loss maintenance ${ }^{(16)}$. In that trial, a modest decrease in the carbohydrate:protein ratio and a modest reduction in the dietary glycaemic index led to an improvement in the maintenance of weight loss. Similar to the mouse experiment, the decreased ratio was achieved by replacing carbohydrates with proteins keeping total fat intake constant between groups. In our study, the intake of total fat differed between groups. If, indeed, the amount of fat rather than the type of fat is of importance, the differences in total fat between groups in our human study may explain the different results.
The findings from the present study are in agreement with previous findings of no substantial associations between intake of PUFA and change in body weight in human populations ${ }^{(17,18)}$. However, in these previous studies intake of $n-6$ PUFA in combination with intake of $n$-3 PUFA was investigated and the background diet was not taken into account.

\section{Conclusion}

In conclusion, in the present study among human participants no consistent associations between the intake of $n$-6 PUFA at a low, medium or high carbohydrate:protein ratio and change in body weight or waist circumference were observed. The inherent difficulties in dietary assessment as well as the differences in exposure levels and exposure contrasts may explain the different findings in our human study and previous animal experiments. However, intakes of $n$ - 6 PUFA, carbohydrates and proteins similar to the animal experiments would imply major changes in dietary habits in the general population. In the general population, the variation in energy intake is primarily accounted for by variation in intake of carbohydrates and fat whereas intake of proteins does not vary substantially. Finally, the different findings in the present human study and in previous animal experiments may arise because the effect of a dietary exposure on body fat mass depends on whether changes in the dietary intake are investigated or whether the habitual dietary intake is investigated. In our human study, habitual intakes of $n-6$ PUFA, carbohydrates and proteins were investigated, contrary to animal experiments where substantial changes in intakes of $n-6$ PUFA, carbohydrates and proteins were investigated.

\section{Acknowledgements}

Financial support: This work was part of the research programme of the Danish Obesity Research Centre (DanORC), which was supported by the Danish Council for Strategic Research (M.U.J., L.M., T.I.A.S., K.K. and K.O., grant number 2101-06-0005) and the research project Hepatic and Adipose Tissue and Functions in the Metabolic Syndrome (HEPADIP), which was supported by the European Commission as an Integrated Project under the 6th Framework Programme (M.U.J., T.I.A.S. and K.O., grant number LSHM-CT-2005-018734). The Danish Council for Strategic Research and the European Commission had no role in the design, analysis or writing of this manuscript. Conflict of interest: None. Authorship: The authors' responsibilities were as follows. M.U.J., L.M., K.K. and K.O.: formulation of research question; M.U.J., L.M., K.K. and K.O.: study design; K.O.: collection of data; C.D. and K.M.D.: statistical analysis of data; M.U.J., L.M., 
C.D., K.M.D., J.H., T.I.A.S., K.K. and K.O.: interpretation of data; and M.U.J.: writing of the manuscript. All authors read and approved the final manuscript. Ethics of human subject participation: The study was conducted according to the guidelines laid down in the Declaration of Helsinki and all procedures involving human subjects were approved by the Copenhagen and Aarhus Ethical Committees. Written informed consent was obtained from all participants.

\section{Supplementary material}

To view supplementary material for this article, please visit http://dx.doi.org/10.1017/S1368980014001578

\section{References}

1. Pischon T, Boeing H, Hoffmann K et al. (2008) General and abdominal adiposity and risk of death in Europe. $N$ Engl $J$ Med 359, 2105-2120.

2. Alvheim AR, Malde MK, Osei-Hyiaman D et al. (2012) Dietary linoleic acid elevates endogenous 2-AG and anandamide and induces obesity. Obesity (Silver Spring) 20, 1984-1994.

3. Simopoulos AP (2002) The importance of the ratio of omega-6/omega-3 essential fatty acids. Biomed Pharmacother 56, 365-379.

4. Blasbalg TL, Hibbeln JR, Ramsden CE et al. (2011) Changes in consumption of omega- 3 and omega- 6 fatty acids in the United States during the 20th century. Am J Clin Nutr $\mathbf{9 3}$, 950-962.

5. Cleary MP, Phillips FC \& Morton RA (1999) Genotype and diet effects in lean and obese Zucker rats fed either safflower or coconut oil diets. Proc Soc Exp Biol Med 220, $153-161$.

6. Massiera F, Saint-Marc P, Seydoux J et al. (2003) Arachidonic acid and prostacyclin signaling promote adipose tissue development: a human health concern? J Lipid Res 44, 271-279.

7. Okuno M, Kajiwara K, Imai S et al. (1997) Perilla oil prevents the excessive growth of visceral adipose tissue in rats by down-regulating adipocyte differentiation. J Nutr 127, $1752-1757$.

8. Matsuo T, Takeuchi H, Suzuki H et al. (2002) Body fat accumulation is greater in rats fed a beef tallow diet than in rats fed a safflower or soybean oil diet. Asia Pac J Clin Nutr 11, 302-308.

9. Madsen L, Pedersen LM, Liaset B et al. (2008) cAMPdependent signaling regulates the adipogenic effect of $n-6$ polyunsaturated fatty acids. J Biol Chem 283, 7196-7205.

10. Tjønneland A, Olsen A, Boll K et al. (2007) Study design, exposure variables, and socioeconomic determinants of participation in Diet, Cancer and Health: a population-based prospective cohort study of 57,053 men and women in Denmark. Scand J Public Health 35, 432-441.

11. Overvad K, Tjønneland A, Haraldsdottir J et al. (1991) Development of a semiquantitative food frequency questionnaire to assess food, energy and nutrient intake in Denmark. Int J Epidemiol 20, 900-905.

12. Tjønneland A, Overvad K, Haraldsdottir J et al. (1991) Validation of a semiquantitative food frequency questionnaire developed in Denmark. Int J Epidemiol 20, 906-912.

13. Møller A \& Saxholt E (1996) Levnedsmiddeltabeller 1996 (Food composition tables 1996). Søborg: National Food Agency (in Danish).

14. van Bakel MM, Slimani N, Feskens EJ et al. (2009) Methodological challenges in the application of the glycemic index in epidemiological studies using data from the European Prospective Investigation into Cancer and Nutrition. J Nutr 139, 568-575.

15. Bigaard J, Spanggaard I, Thomsen BL et al. (2005) Selfreported and technician-measured waist circumferences differ in middle-aged men and women. I Nutr 135 , 2263-2270.

16. Larsen TM, Dalskov SM, van Baack M et al. (2010) Diets with high or low protein content and glycemic index for weight-loss maintenance. $N$ Engl J Med 363 , 2102-2113.

17. Field AE, Willett WC, Lissner L et al. (2007) Dietary fat and weight gain among women in the Nurses' Health Study. Obesity (Silver Spring) 15, 967-976.

18. Forouhi NG, Sharp SJ, Du H et al. (2009) Dietary fat intake and subsequent weight change in adults: results from the European Prospective Investigation into Cancer and Nutrition cohorts. Am J Clin Nutr 90, 1632-1641. 